\title{
Production and localization of restrictocin in Aspergillus restrictus
}

\author{
Tristan T. BRaNDHORST and William R. KENEALY* \\ Department of Biochemistry, 420 Henry Mall, College of Agricultural and Life Sciences, University of Wisconsin, \\ Madison, Wisconsin 53706, USA
}

(Received 21 January 1992; accepted 14 February 1992)

\begin{abstract}
The production and secretion of restrictocin (a cytotoxin that cleaves ribosomal RNA) by cultures of the fungus Aspergillus restrictus was investigated. Previous studies have indicated that restrictocin production in liquid culture coincides with the appearance of differentiated cell structures. A study of the correlation between the appearance of differentiated structures and restrictocin production was conducted with $A$. restrictus grown on agar medium. Restrictocin was found to be associated with the cell mass of the agar-grown culture (in contrast to liquid cultures), and was first observed when aerial hyphae emerged. Restrictocin levels increased until the time of conidiation, after which they fell off sharply. No restrictocin could be found in the agar medium. The presence of restrictocin upon and within various cell structures was determined by immunofluorescent laser microscopy. This study showed that restrictocin became localized to the conidiophores and phialides during the process of conidiation. Prior to this, restrictocin was found within the hyphae in localized concentrations that may correspond to secretory vesicles.
\end{abstract}

\section{Introduction}

Aspergillus restrictus produces and secretes restrictocin, a small basic protein $\left(M_{\mathrm{r}} 17000\right)$ which is an inhibitor of protein synthesis. Restrictocin is a ribosome-inactivating protein (RIP) and closely resembles the RIPs mitogillin (also from $A$. restrictus) and $\alpha$-sarcin (from Aspergillus giganteus) (Conde et al., 1978). These RIPs have been investigated for their potential as anti-tumour agents (Goldin et al., 1966; Olson et al., 1963, 1965; Roga et al., 1971). Human clinical trials had to be terminated, however, due to non-specific toxicity (Wool, 1984).

The toxicity of restrictocin is due to its ability to enter cells and catalytically hydrolyse a specific phosphodiester bond in the 28S rRNA subunit (Fando et al., 1985; Schindler \& Davies, 1977). Because this action is catalytic, one active molecule of restrictocin would theoretically be enough to result in the death of a cell. This same ribonuclease activity has made restrictocin a useful tool in the study of ribosome structure and ribosome-protein interactions (Wool, 1984). The site of action of restrictocin is conserved in the ribosomes of all eukaryotes and prokaryotes tested thus far, including ribosomes of Aspergillus spp. The ribosomes of $A$. giganteus are subject to cleavage by its own $\alpha$-sarcin RIP

*Author for correspondence. Tel. (608) 262 4392; fax (608) 2623453.

Abbreviation: RIP, ribosome-inactivating protein. in vitro (Miller \& Bodley, 1988). This raises the question of what keeps the RIP-producing Aspergillus spp. from succumbing to their own toxins. Lamy \& Davies (1991) suggested that pro-restrictocin might be inert until the protein is processed during secretion.

It was recently reported that the Asp Fl antigen produced by Aspergillus fumigatus is a RIP similar to mitogillin and restrictocin (Arruda et al., 1990). Asp F1 raises an IgE response in allergic bronchopulmonary aspergillosis that might be expected to augment the cytotoxic effect of the two A. fumigatus toxins, fumagillin and gliotoxin. Asp F1 has a $95 \%$ homology to restrictocin and mitogillin (Arruda et al., 1991). These RIPs have all been shown to have an inhibitory effect on ongoing lymphocyte responses to toxins administered in vivo (Hutcheson et al., 1991). In liquid culture, both $A$. restrictus and $\boldsymbol{A}$. fumigatus produce RIPs only after significant accumulation of cell mass. What induces the fungi to produce RIPs is unknown.

Despite the amount of research performed thus far to determine the structure of restrictocin, the mechanism by which it gains entry into certain cell types, and its exact mode of action, no data have been gathered on the role of restrictocin in the natural life cycle of $A$. restrictus. Previous studies involving restrictocin have invariably employed agitated liquid culture techniques to produce the cytotoxin, and were carried out with protein purified from the broth, but fungi such as $A$. restrictus are often 
found in water-stressed environments rather than immersed in medium (Kozakiewicz, 1989). Is restrictocin even made when the fungus is grown on solid medium? If so, does it build up anywhere on the surface of the fungus without a liquid environment to rinse it away?

A correlation between the appearance of restrictocin and the appearance of certain differentiated structures (conidiophores) in liquid medium has previously been observed in this laboratory (Yang \& Kenealy, 1992). Growing A. restrictus on solid medium is better suited to a study of the correlation between differentiated structures and restrictocin production as these cell structures do not develop properly in a liquid environment. This paper focuses on the production of restrictocin by cells grown on solid medium and on the localization of restrictocin in these cultures.

\section{Methods}

Culture of organism. A. restrictus (strain NRRL 2869) was maintained on complete fungal medium agar plates (Cove, 1966). Conidia from the agar plates were suspended in Luria broth (LB) (Maniatis et al., 1982) at a concentration of $2 \times 10^{7}$ conidia $\mathrm{ml}^{-1}$. The conidial suspension was frozen at $-20^{\circ} \mathrm{C}$ in $0.4 \mathrm{ml}$ aliquots. Complete fungal medium agar plates were overlaid with sterilized membranes of nitrocellulose (NitroME, $0.45 \mu \mathrm{m}$, from MSI). To generate plate cultures, $0.4 \mathrm{ml}$ of conidial suspension was spread across the suface of the nitrocellulose membrane and allowed to soak in. Plates were inverted and incubated at $37^{\circ} \mathrm{C}$. For liquid cultures, $0.4 \mathrm{ml}$ of conidial suspension was inoculated into $25 \mathrm{ml}$ of complete fungal liquid medium. Flasks were shaken at 200 r.p.m. at $37^{\circ} \mathrm{C}$.

Extraction and analysis of protein from cultures. Cell mass was harvested by peeling the nitrocellulose membranes, to which the fungus adhered, off the agar medium. The nitrocellulose membranes and the adhering $A$. restrictus were then crushed to a fine powder under liquid nitrogen with a pre-cooled mortar and pestle. This powder was suspended in $5 \mathrm{ml} \mathrm{1} \%(\mathrm{w} / \mathrm{v})$ SDS and homogenized with a Polytron homogenizer for $2 \mathrm{~min}$. The homogenate was heated in a boiling water bath for $5 \mathrm{~min}$ and then centrifuged in a clinical centrifuge for $15 \mathrm{~min}$. The supernatant was used in further assays.

Cell mass from liquid cultures was harvested by collection onto type 1 Whatman filters. Cells were rinsed once with water and then frozen, crushed, homogenized, heated and centrifuged as above.

Total protein was quantified by the bicinchoninic acid (BCA) assay (Pierce). Bovine serum albumin was used as a standard. The absorbance at $562 \mathrm{~nm}$ was read on a micro-plate reader (Bio-Tek) and the assays were performed in triplicate.

Extraction of protein from agar. Protein was extracted from the agar plates by adding 1 vol. of $2 \%(w / v)$ SDS to 1 vol. of the melted agar, freezing the agar at $-20^{\circ} \mathrm{C}$, melting the frozen agar at room temperature, and centrifuging at $1500 \mathrm{~g}$ for $60 \mathrm{~min}$ in a clinical centrifuge. The agar pelleted to the bottom under these conditions. Proteins were concentrated from the supernatant by precipitation with $50 \%$ (v/v) acetone (Lamy \& Davies, 1991).

Detection of restriction. Samples from the protein-containing solutions above were mixed with 1 vol. of sample buffer $(60 \mathrm{~mm}-\mathrm{Tris} / \mathrm{HCl}$, $\mathrm{pH} 6.8,60 \%, \mathrm{v} / \mathrm{v}$, glycerol, $2 \%, \mathrm{w} / \mathrm{v}, \mathrm{SDS}, 5 \%, \mathrm{v} / \mathrm{v}, \boldsymbol{\beta}$-mercaptoethanol, $0.001 \%$ bromophenol blue), heated in a boiling water bath for $10 \mathrm{~min}$ and run on a $12 \%(\mathrm{w} / \mathrm{v})$ polyacrylamide SDS gel (Laemmli, 1970). The gel was electro-blotted onto nitrocellulose using a semi-dry electrophor- etic transfer unit (Gelman). The blot was stained with amido black and blocked with blotto (Johnson et al., 1984). The indirect, alkalinephosphatase-linked detection system described by Yang \& Kenealy (1992) was used for the detection of restrictocin on this blot. The BCIP/NBT substrate system from Kirkegaard and Perry Laboratories was used for the colour formation reaction. The detection limit of this system was determined to be $10 \mathrm{ng}$ of restrictocin protein.

Estimation of total restrictocin. Each sample for restrictocin analysis was serially diluted with $1 \%$ SDS to yield concentrations of $1 \times, 0.5 \times$ and $0.1 \times$. A purified restrictocin control sample (Yang \& Kenealy, 1992) was also diluted to provide samples containing $500,400,300,200$, $100,75,50,25$ and $10 \mathrm{ng}$, respectively. Experimental and control dilutions were run together on a $12 \%$ polyacrylamide SDS gel and Western blotted as described above. Blots were scanned on a Hewlett Packard Scanjet Plus and the densities of the bands were estimated using the Deskscan and NIH Image 1.40 programs on a Macintosh II. The densities of the control bands were used to construct a standard curve to which the sample dilutions could be compared. The resulting estimations of the restrictocin content of each sample dilution were averaged to determine total restrictocin. The results of two series of blots were averaged to provide the final estimations of total restrictocin.

Morphological observations. Observations of fungal morphology and differentiated structures were made throughout the incubation period by visual inspection (in the case of prominent surface features such as aerial hyphae) and phase contrast microscopy (in the case of developing conidial structures). A Zeiss Axioskop microscope with a $40 \times$ objective was used for these observations.

Confocal laser fluorescence microscopy. Samples of plate-grown $A$. restrictus were taken at various times by cutting out and removing $0.5 \mathrm{~cm}$ squares of the nitrocellulose support. Samples $(1 \mathrm{ml})$ of flaskcultured $A$. restrictus were taken at similar times and centrifuged to separate hyphae from medium. Samples of hyphae were either fixed with $95 \%$ ethanol, fixed with Carnoy's solution $(60 \%$ ethanol, $30 \%$ chloroform, $10 \%$ acetic acid, by vol.), or left unfixed and treated with phosphate-buffered saline $+0.05 \%$ Tween 20 (PBST) to wet the hydrophobic cell structures. The samples were next washed with $1 \%$ (v/v) goat serum in phosphate-buffered saline (PBS), followed by exposure to polyclonal anti-restrictocin antibodies from rabbit (Yang \& Kenealy, 1992) diluted 1:400 in PBS for $20 \mathrm{~min}$. After three washes with PBST, samples were exposed for $20 \mathrm{~min}$ to FITC-labelled antibodies against rabbit IgG (raised in goat; Sigma), diluted to $1.25 \mu \mathrm{g} \mathrm{m}^{-1}$ in PBS. Excess antibodies were washed away with two PBST rinses and two PBS rinses.

Samples were teased apart with forceps prior to the addition of a coverslip. Hyphae and conidiophores were observed using a Nikon Optiphot microscope coupled with a Bio-Rad MRC 600 confocal laser scanning system. An argon ion laser from Ion Laser Technology, with a model 5425 laser head, was used for illumination. Observations were made using a Nikon $60 \times$ oil immersion lens. For all observations the laser was used at half power with a 1.0 neutral density filter and a confocal aperture setting of 6 . The scanner was set for maximum gain, automated black level, and a low signal enhancement setting of 1 . Images were stored on a Panasonic 3031F optical memory disk recorder.

\section{Results}

\section{Growth and morphology of $A$. restrictus}

$A$. restrictus cell mass on agar plates may be detected by visual inspection as early as $7 \mathrm{~h}$ after inoculation. This 


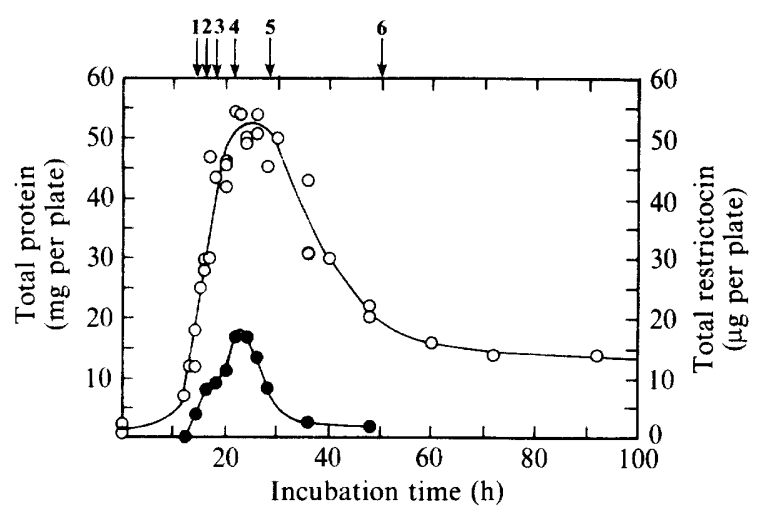

Fig. 1. Protein and restrictocin accumulation by $A$. restrictus cultured on agar. $O$, Total protein;, total restrictocin. Data plotted are the results from three separate experiments. Numbers indicate first observations of morphological development: (1) appearance of aerial hyphae; (2) swelling of hyphal tips; (3) formation of phialides; (4) conidiation; (5) development of blue pigmentation; (6) hyphae empty of cytosol.

cell mass thickens with time until the culture takes on a blue coloration. Measurements of SDS-extractable protein (Fig. 1) taken throughout the life cycle of $A$. restrictus show that protein levels increase with the initial cell mass and then decrease rapidly following the colour change. A significant proportion of the dry mass of the fungus after the colour change is composed of empty hyphal tubes. Protein determinations were used in lieu of dry weight determinations to follow the growth and subsequent degeneration of $A$. restrictus grown on agar medium.

When propagated at $37^{\circ} \mathrm{C}$ on a nitrocellulose membrane overlaying complete medium agar, the fungus completes growth and conidiation in a little over $2 \mathrm{~d}$. Microscopy observations of $A$. restrictus were made during these incubations. In Fig. 1, a record of the morphological developments has been placed in the context of the total protein curve for comparison. Aerial hyphae were first visible after $14 \mathrm{~h}$ growth. The tips of these hyphae become swollen and spherical by $16 \mathrm{~h}$ growth and sterigmata (phialides) (Thom \& Raper, 1945) become evident at $18 \mathrm{~h}$. Conidia began to form upon the phialides at approximately $22 \mathrm{~h}$, but the blue coloration associated with mature conidia did not develop upon the plates until after $28 \mathrm{~h}$. Cytosol appeared to be absent from all structures except the conidia by $50 \mathrm{~h}$ after inoculation.

The quantity of SDS-extractable protein present was at its highest as the first conidia were formed. Total protein levels dropped rapidly from the time of conidiation until about $50 \mathrm{~h}$ after inoculation, after which protein levels diminished only a little.

\section{Crude distribution of restrictocin}

Since restrictocin is primarily found free in the medium of $A$. restrictus liquid cultures, we wished to determine whether restrictocin was secreted by our cultures into the agar medium. To this end, a technique by which proteins could be extracted from agar medium was developed. Purified restrictocin (100, 50 and $10 \mathrm{ng}$ quantities) was added either to $10 \mathrm{ml}$ samples of fresh molten agar medium or to $10 \mathrm{ml}$ samples of liquid medium (as controls) and extracted by the protocol outlined in Methods. An immunoblot comparison of the concentrated protein from these controls (Fig. 2, lanes 1-3) and extracts (Fig. 2, lanes 4-6) showed that the amount of restrictocin recovered from the agar was almost equal to that recovered from the liquid medium.

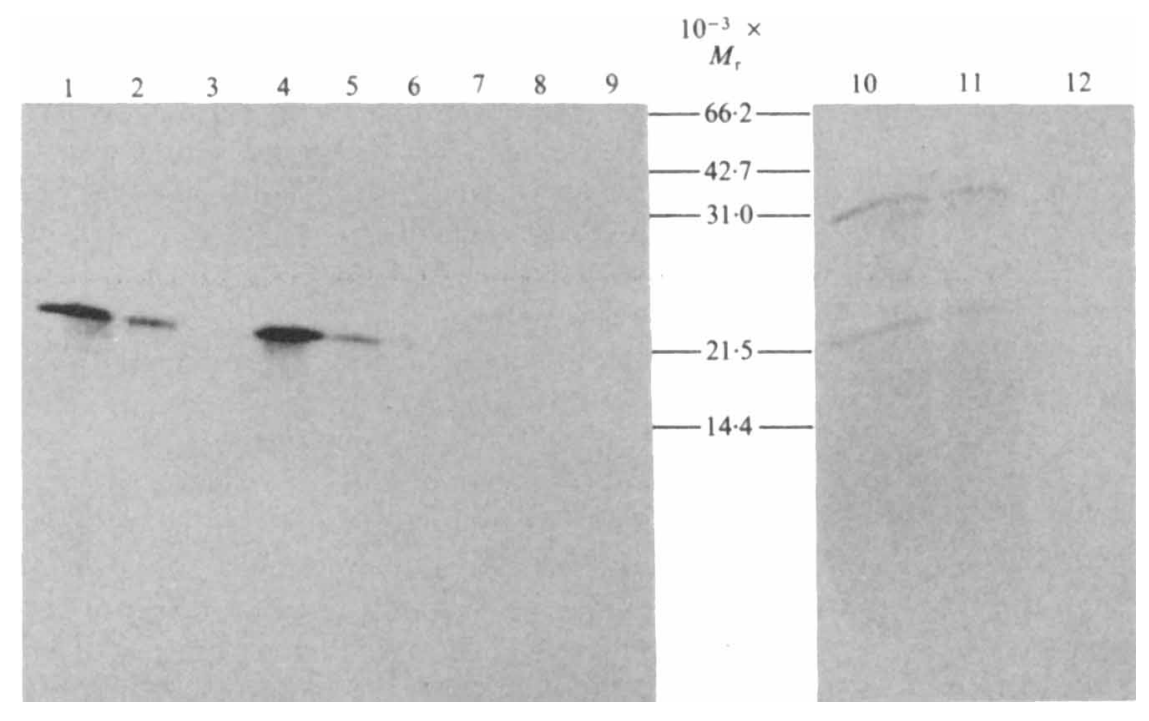

Fig. 2. Immunoblot of restrictocin extracted from agar and cell material. Lanes $1-3$, restrictocin reference lanes containing 100,50 and $10 \mathrm{ng}$ of restrictocin, respectively. Lanes $4-6,100,50$ and $10 \mathrm{ng}$ of restrictocin that was added to agar and then re-extracted. Lanes $7-9$, total protein extracted from $A$. restrictus agar cultures after 16,28 and $36 \mathrm{~h}$ incubation. Lanes 10-12, total protein from $A$. restrictus mycelium after $28 \mathrm{~h}$ incubation; lanes contain $100 \mu \mathrm{l}$ of $1 \times, 0.5 \times$ and $0.1 \times$ dilutions of $A$. restrictus cell extract. Standard proteins $\left(M_{\mathrm{r}}\right.$ values indicated) were run on the same gel. 
Proteins were extracted from the agar of $A$. restrictus cultures at 16, 28 and $36 \mathrm{~h}$ and concentrated as above. The concentrated proteins were subjected to PAGE and Western blotted onto nitrocellulose. Amido black staining, which has a detection limit of approximately $1 \mu \mathrm{g}$ protein, made visible several bands of secreted fungal protein. The intensity of these bands increased in samples taken from cultures that were incubated for longer periods. One of these, probably glucoamylase, migrated at an $M_{\mathrm{r}}$ of 80000-90000, confirming that large fungal proteins can pass through the nitrocellulose membrane. No bands corresponding to restrictocin were revealed by amido black staining.

No restrictocin could be detected in the agar extract using the immunoblot technique (Fig. 2, lanes 7-9). The immunoconjugate detection system employed by our laboratory can detect as little as $10 \mathrm{ng}$ of restrictocin. Since $A$. restrictus produces over $10 \mu \mathrm{g}$ of restrictocin per plate, less than $0.1 \%$ of the total restrictocin is able to pass into the medium.

Total restrictocin was estimated from the densities of bands on a Western blot. Dilutions of sample protein extracts (Fig. 2, lanes 10-12) were assayed in this manner and known amounts of restrictocin (Fig. 2, lanes 1-3) were run on the same gels as the samples. Restrictocin standards and sample dilutions were selected so as to be within the range where band intensity is linear with concentration.

Total restrictocin from plate cultures was plotted versus time in Fig. 1 for comparison with total protein and fungal morphology. It may be noted from this graph that restrictocin was first detected at the same time that the fungus puts forth aerial hyphae. The increase in total restrictocin paralleled total protein from this point until the time of conidiation, after which restrictocin was apparently degraded by the fungus.

In Fig. 3, the total protein associated with cell mass derived from liquid cultures of $A$. restrictus was compared to the restrictocin present in both cell mass and liquid medium. The culture conditions were set up to be comparable to our plate cultures (restrictocin had been produced in higher concentrations in media where its production was optimized). More restrictocin was produced per milligram of total cellular protein in liquid culture and most of the restrictocin was found in the medium. Less restrictocin remained associated with the cell mass under liquid culture conditions.

\section{Localization of restrictocin}

Several methods of preparing the cell material were used. Only minute differences were observed between samples that were fixed with $95 \%$ ethanol and unfixed samples. Ethanol fixation was found to enhance the sharpness and

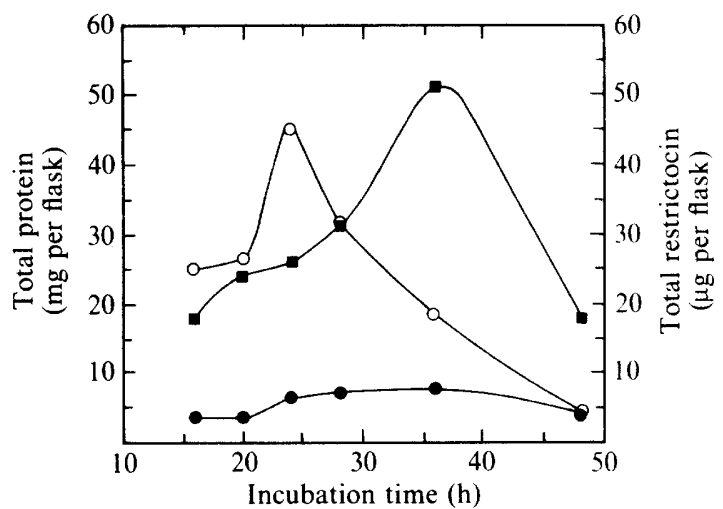

Fig. 3. Protein and restrictocin accumulation by $A$. restrictus cultured in liquid medium. $O$, Total protein; $\boldsymbol{O}$, cell-associated restrictocin; $\mathbf{\square}$, restrictocin from supernatant. Data from a representative experiment.

definition of fluorescent images to some small degree, but the characteristics of fluorescence distribution were unaffected. The use of a fixative such as Carnoy's solution was necessary to ensure penetration of immunological reagents to the inside of the hyphae.

Controls which examined the level of fluorescence due to background and non-specific binding activity were run with the samples. In one set of sub-samples, the fluorescein-conjugated second antibody was omitted from the protocol so that native fluorescence levels could be observed. No such background fluorescence was detected under the conditions used. In another set of subsamples, the anti-restrictocin rabbit serum was replaced with normal rabbit serum in order to account for nonspecific absorption of rabbit antibodies or an affinity of the secondary antibody for the cell material. A low level of non-specific fluorescence was observed in these subsamples (Fig. $4 a, b$ ).

Fig. 1 indicates that restrictocin was not detectable until $14 \mathrm{~h}$ of incubation. Our immunofluorescent microscopy survey of restrictocin in cultures of $A$. restrictus began at this time of incubation. Between 14 and $18 \mathrm{~h}$, very small quantities of restrictocin were observed on the surface of the hyphae, homogeneously distributed (data not shown). Samples treated with Carnoy's solution showed highly fluorescent spots after $16 \mathrm{~h}$ (Fig. 4c). As the conidiophores developed mature phialides around $22 \mathrm{~h}$, many of them displayed very intense fluorescence, indicating elevated levels of restrictocin on their surfaces. At its peak, the intensity of fluorescence upon the surface of the phialides was dramatic (Fig. 4d). It appeared to coat the outsides of the phialides and the surfaces of the conidiophores that lie between them, yet, interestingly, it did not coat the immature conidia that bud off the end of the phialides (Fig. 4e). During the time that the main fluorescence signal was seen on the conidiophores, there was a commensurate lessening of 

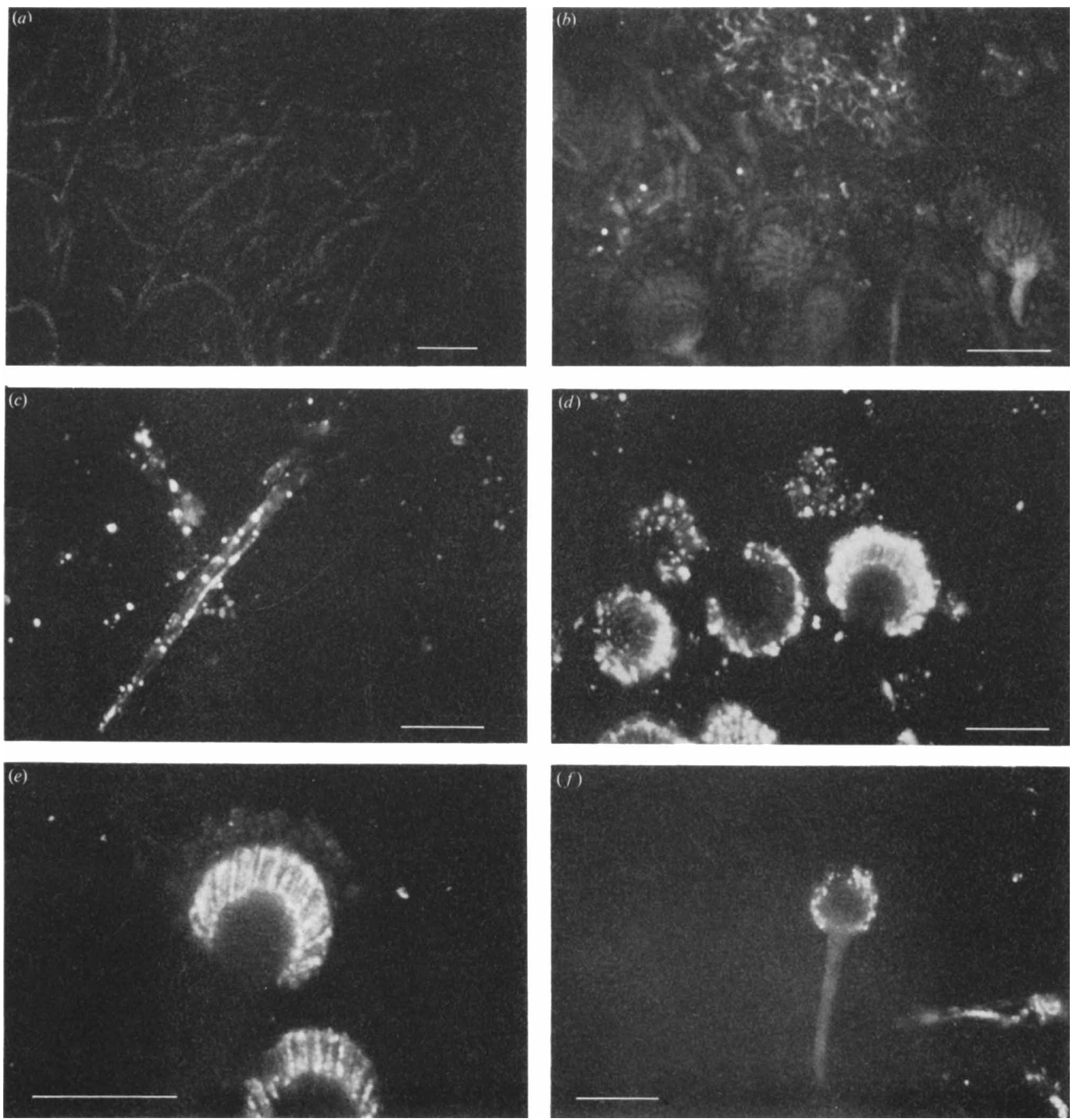

Fig. 4. Immunofluorescent photomicrograpns of $\boldsymbol{A}$. restrictus. All cell material was treated as described in Methods. (a) Hyphae fixed at $16 \mathrm{~h}$ with ethanol (negative control, normal rabbit serum); (b) hyphae and conidiophores fixed at $26 \mathrm{~h}$ with Carnoy's solution (negative control, normal rabbit serum); (c) hyphae fixed at $16 \mathrm{~h}$ with Carnoy's solution; $(d)$ conidiophores fixed at $26 \mathrm{~h}$ with ethanol; $(e)$ conidiophores fixed at $28 \mathrm{~h}$ with Carnoy's solution; $(f)$ unfixed conidiophore after $36 \mathrm{~h}$ incubation. Bars, $5 \mu \mathrm{m}$.

fluorescence in and on the (undifferentiated) hyphae. Samples treated with Carnoy's solution showed fewer and fainter 'hot spots' within the hyphae after $24 \mathrm{~h}$. After $26 \mathrm{~h}$, the intensity of the fluorescence on the conidiophores began to diminish steadily (Fig. $4 f$ ). At $48 \mathrm{~h}$, it could barely be distinguished from the non-specific background. All these results were highly reproducible and at no point during our experiments was restrictocin observed upon the surfaces of conidia.

Samples taken from liquid cultures were also observed by immunofluorescent microscopy. Despite the presence of structures resembling incompletely formed conidio- 
phores, none of these cell samples exhibited any of the intense, localized fluorescence seen in the samples from solid medium.

\section{Discussion}

Our results show conclusively that restrictocin is made by cultures of $A$. restrictus grown on solid medium. Moreover, there appears to be a specific cellular localization of the restrictocin to the phialides. These results are in sharp contrast to those found when liquid cultures of $A$. restrictus were examined. In liquid culture little restrictocin is found associated with cell material and the bulk of the restrictocin is found in the medium.

The production of restrictocin in both liquid (Yang \& Kenealy, 1992) and solid medium is concurrent with differentiation leading to conidia. Whether this is due to a cause-effect relationship between the two phenomena, or to an induction pathway that both share, is as yet unknown. We have shown that for agar-grown $A$. restrictus the signal for conidiation and restrictocin production is quite reproducible. The signal is probably related to the exhaustion of a nutrient or the production of a metabolite and in small areas of an agar plate this can occur quite rapidly. While it is possible that restrictocin has a role in the process of conidiation it is equally possible that it serves some protective or invasive function for the fungus in its natural environment.

In our observations, restrictocin is present on the phialides as they mature. It is also at this time that the amount of restrictocin detectable is greatest. In many species of Aspergillus, the first conidia emerge through the outer wall of the phialides, forming fresh cell wall within collarettes at the tip of the phialides (Trinci et al., 1968; Tsukahara, 1970; Oliver, 1972; Ghorse \& Edwards, 1973; Hanlin, 1976). Our results suggest that a similar mechanism operates in $A$. restrictus, such that the conidial walls are derived from an apical plug rather than being continuous with the phialide. As can be seen in Fig. 4(e), the walls of the emerging conidia are not coated with restrictocin, unlike the phialides from which they issued. This could be an indication that restrictocin secretion ceases as the first conidia differentiate, or that the direction of restrictocin secretion is specific to the phialides only.

An advantage in producing restrictocin just before the stage of conidiation might lay in a toxicity to predators of the fungus, $A$. restrictus having been reported in a close association with two different species of beetles (Kozakiewicz, 1989). Restrictocin could thus discourage predation upon conidiophores until their development was complete. It could be that restrictocin inactivates cells within the digestive systems of insects that feed upon the conidiophore, allowing the spores to be passed intact by the insect or even allowing them to germinate within the insect, killing it and growing upon its remains.

Another advantage for an RIP-producing culture could be an enhancement of its pathogenicity to vertebrate tissues, as noted by Arruda et al. (1991). Several factors suggest that at least the initial pathogenic invasion of tissue by fungal hyphae does not require restrictocin. Restrictocin is not localized to the surface of $A$. restrictus conidia and is not found associated with conidia analysed by Western tlotting so, prior to germination, it could not be a factor in the infection of tissue by air-borne conidia. Also, RIPs are not produced by $A$. restrictus or $A$. fumigatus until there is an appreciable build-up of cell mass, as is common with secondary metabolites in the filamentous fungi (Arrunda et al., 1991). It is certainly possible that the reaction of a host's immune system to the fungus could trigger the sequence of conidiation and restrictocin production, but we did not perform experiments to determine this.

The liquid cultures of $A$. restrictus differ considerably from those grown on solid medium. The cultures in liquid usually have 'less-concentrated' cell mass and exhaustion of nutrient or the build-up of products takes considerably longer. In fact, the time needed to attain maximum cell mass, coupled with the reduced tendency towards conidiation in liquid medium, makes the differentiation leading to conidiation less reproducible. However, even though the differentiation leading to conidiation is less apparent in liquid culture it does take place and it is concurrent with restrictocin production (Yang \& Kenealy, 1992).

In our shake-flask experiments, restrictocin was not found in association with differentiated cells, yet the levels of restrictocin production are higher than in plate culture. This could mean that restrictocin production is highest just prior to conidiation, a state that can be quite long for fungi cultivated in the submerged state. Another possibility is that the fungus strives to replace restrictocin upon the surface of the conidiophore as it is washed off into the liquid medium. Perhaps some accumulation of restrictocin upon the conidiophores of $A$. restrictus is required before conidiation can properly commence.

Restrictocin was not found in the agar of our solid medium cultures as might be expected from the liquid culture results. Since fungal-produced proteins were found in the agar it seems logical that any restrictocin secreted by the hyphae either remained in association with those hyphae or became bound to the nitrocellulose membrane. We did not determine whether nitrocellulose preferentially bound restrictocin. Association of the restrictocin with the hyphae could be fairly weak since in liquid medium the restrictocin is found separate from the cells. It may be that restrictocin adheres to the surface of 
the fungus upon secretion, or that it is secreted only by structures such as conidiophores that are held away from the surface of the agar.

Immunofluorescent observations of cells fixed with Carnoy's solution show that 'hot spots' of restrictocin concentration exist within hyphae that are $16-26 \mathrm{~h}$ old. These 'hot spots' could correspond to a build-up of restrictocin in the endoplasmic reticulum and/or secretory vesicles (Fig. 4c). If this were the case, it would indicate that restrictocin was being synthesized at a distance from the point of secretion/localization. Some aspect of restrictocin, perhaps the leader sequence or a short 'pro' sequence (Yang \& Kenealy, 1992), would have to target it to its final destination upon the surface of the phialides. We are performing further studies to elucidate any role played by $\mathrm{N}$-terminal leader sequences in restrictocin targeting. We are also examining the ability of these extensions to prevent self-toxification in the fungus as predicted by Lamy \& Davies (1991).

We thank the IMR facility for the use of their confocal laser imaging equipment. The IMR in Madison is funded as an NIH Biomedical Research Technology Resource (RR570). This research was supported by the College of Agricultural and Life Sciences and by a Steenbock Career Development Award, University of Wisconsin, Department of Biochemistry. T. Brandhorst was supported in part by a Cell and Molecular Biology Training Grant, NIH.

\section{References}

Arruda, L. K., Platts-Mills, T. A. E., Fox, J. W. \& Chapman, M. D. (1990). Aspergillus fumigatus allergen I, a major IgE-binding protein, is a member of the mitogillin family of cytotoxins. Journal of Experimental Medicine 172, 1529-1532.

Arruda, L. K., Good, M., Platts-Mills, T. A. E. \& Chapman, M. D. (1991). Asp FI, a major Aspergillus fumigatus allergen: homology to the cytotoxin mitogillin and measurements in spore, mycelial and culture filtrate extracts. Journal of Allergic and Clinical Immunology 87, 168.

Conde, F. P., Fernandez-Puentes, C., Montero, M. T. V. \& VAZQueZ, D. (1978). Protein toxins that catalytically inactivate ribosomes from eukaryotic microorganisms. FEMS Microbiology Letters 4, 349-355.

Cove, D. J. (1966). The induction and repression of nitrate reductase in the fungus Aspergillus nidulans. Biochimica et Biophysica Acta 113, 5156.

Fando, J. L., Alaba, I., Escarmis, C., Fernandez-luna, J. L., Mendez, E. \& Salinas, M. (1985). The mode of action of restrictocin and mitogillin on eukaryotic ribosomes. European Journal of Biochemistry 149, 29-34.
GHORSE, W. C. \& EDWARDs, M. R. (1973). Ultrastructure of Aspergillus fumigatus conidia development and maturation. Protoplasma 76, 4959.

Goldin, A., Serpick, A. A. \& Mantel, N. (1966). Experimental screening procedures and clinical predictability value. Cancer Chemotherapy Reports 50, 173-218.

HaNliN, R. T. (1976). Phialide and conidium development in Aspergillus clavatus. American Journal of Botany 63, 144-155.

Hutcheson, P. S., Sligh, J. M., Mueller, K. R., ArRuda, L. K. \& SLAviN, R. G. (1991). Lymphocyte proliferative responses to purified antigens of Aspergillus. Journal of Allergic Clinical Immunology 87, 196.

Johnson, D. A., Gautsch, J. W., Sportsman, J. R. \& Elder, J. H. (1984). Improved technique utilizing nonfat dry milk for analysis of proteins and nucleic acids transferred to nitrocellulose. Gene Analysis Techniques 1, 3-8.

KozAKIEWICZ, Z. (1989). Aspergillus species on stored products. Mycological Papers 161, CAB International Mycological Institute, UK.

LAEMMLI, U. K. (1970). Cleavage of structural proteins during the assembly of the head of bacteriophage T4. Nature, London 227, 680685.

LAMY, B. \& DAviES, J. (1991). Isolation and nucleotide sequence of the Aspergillus restrictus gene coding for the ribonucleolytic toxin restrictocin and its expression in Aspergillus nidulans: the leader sequence protects producing strains from suicide. Nucleic Acids Research 19, 1001-1006.

Maniatis, T., Fritsch, E. F. \& SambrooK, J. (1982). Propagation and maintenance of bacterial strains and viruses. In Molecular Cloning : $A$ Laboratory Manual, p. 68. Cold Spring Harbor, NY: Cold Spring Harbor Laboratory.

MilleR, S. P. \& BoDley, J. W. (1988). The ribosomes of Aspergillus giganteus are sensitive to the cytotoxic action of $\alpha$-sarcin. FEBS Letters 229, 388-390.

OlIVER, P. T. P. (1972). Conidiophore and spore development in Aspergillus nidulans. Journal of General Microbiology 73, 45-54.

Olson, B. H., Harvey, C. L. \& Junek, A. J. (1963). US patent \# 3104208 .

Olson, B. H., Jennings, J. C., Roga, V., Junek, A. J. \& SChuURmans, D. M. (1965). Alpha sarcin, a new antitumor agent. II. Fermentation and antitumor spectrum. Applied Microbiology 13, 322-326.

Roga, V., Hedeman, L. P. \& Olson, B. H. (1971). Evaluation of mitogillin (NSC-69529) in the treatment of naturally occurring canine neoplasms. Cancer Chemotherapy Reports 55, 101-113.

SChINDleR, D. G. \& Davies, J. E. (1977). Specific cleavage of ribosomal RNA caused by alpha sarcin. Nucleic Acids Research 4 , 1097-1110

THOM, C. \& RAPER, K. B. (1945). A Manual of the Aspergilli. Baltimore: Williams and Wilkins.

Trinci, A. P. J., Peat, A. \& Banbury, G. H. (1968). Fine structure of phialide and conidiospore development in Aspergillus giganteus. Annals of Botany 32, 241-249.

TsukaHARA, T. (1970). Electron microscopy of conidiospore formation in Aspergillus niger. Sabouraudia 8, 93-97.

WoOL, I. G. (1984). The mechanism of the action of the cytotoxic nuclease $\alpha$-sarcin and its use to analyse ribosome structure. Trends in Biochemical Sciences 9, 14-17.

YANG, R. \& KENEALY, W. R. (1992). Regulation of restrictocin production in Aspergillus restrictus. Journal of General Microbiology 138, 1421-1427. 